Research Paper

\title{
Andrographolide Enhances TRAIL-Induced Apoptosis via p53-Mediated Death Receptors Up-Regulation and Suppression of the NF-KB Pathway in Bladder Cancer
}

\section{Cells}

\author{
Yuyou Deng1,2, Ran Bi1,2, Haoran Guo², Jiaxin Yang2, Yujun Du³, Chunxi Wang1 ${ }^{\bowtie}$, and Wei Wei2 ${ }^{\bowtie}$ \\ 1. Department of Urology, The First Hospital of Jilin University, Changchun, Jilin Province 130021, China. \\ 2. Institute of Virology and AIDS Research, The First Hospital of Jilin University, Changchun, Jilin Province 130021, China. \\ 3. Department of Nephrology, First Hospital of Jilin University, Changchun, Jilin Province 130021, China. \\ \ Corresponding authors: Wei Wei: wwei6@jlu.edu.cn; Chunxi Wang: chunxiwang1993@163.com \\ (c) Ivyspring International Publisher. This is an open access article distributed under the terms of the Creative Commons Attribution (CC BY-NC) license \\ (https://creativecommons.org/licenses/by-nc/4.0/). See http://ivyspring.com/terms for full terms and conditions.
}

Received: 2018.10.22; Accepted: 2018.12.20; Published: 2019.01.24

\begin{abstract}
Tumor necrosis factor-related apoptosis-inducing ligand (TRAIL) is an effective chemotherapeutic agent that specifically impairs cancer cells while sparing normal cells; however, some cancer cells develop resistance to TRAIL. Here, we identified Andrographolide, a diterpenoid lactone derived from a traditional herbal medicine Andrographis paniculata, as an ideal sensitizer for TRAIL to overcome bladder cancer. Our results showed that combination treatment of Andro and TRAIL retarded growth, attenuated proliferation, decreased colony formation, inhibited migration and promoted caspases-mediated apoptosis in T24 cells. Additionally, the sensitization by Andro is achieved through up-regulation of death receptors (DR4 and DR5) of TRAIL in a p53-dependent manner. Crucially, Andro is also capable of inactivating NF-KB signaling pathway via transcriptional down-regulation $p 65 /$ RelA, which is further contributed to enhancement of TRAIL-mediated cytotoxicity. These results indicated that non-toxic doses of Andrographolide sensitized bladder cancer cells to TRAIL-mediated apoptosis, suggesting it as an effective therapeutic agent for TRAIL resistant human bladder cancers.
\end{abstract}

Key words: andrographolide, bladder cancer, death receptors, TRAIL, NF-KB

\section{Introduction}

Bladder cancer ranks as the $9^{\text {th }}$ most frequently diagnosed cancer and is $13^{\text {th }}$ in terms of deaths worldwide since 2012 [1]. Although accumulating studies show that targeted drugs can improve patient survival rates, first-line molecular-targeted drugs (sorafenib and gefitinib) exhibit only limited therapeutic effects in several clinical trials and can cause severe side effects $[2,3]$. Therefore, there is an increasing demand to explore effective strategies for bladder urothelial carcinoma (BLCA) treatment.

Tumor necrosis factor-related apoptosis-inducing ligand (TRAIL), has been reported as a candidate for cancer treatment due to its selective inducement of apoptosis in cancer cells while sparing normal cells [4, 5]. TRAIL specifically binds to cell-death receptors
DR4 or DR5 (also named tumor necrosis factor receptor superfamily member 10 TNFRSF10A and TNFRSF10B, respectively), resulting in trimerization of the receptor and clustering of the intracellular death domain of the receptor and leading to death-inducing signaling complex formation $[6,7]$. Trimerization of the death domains leads to the recruitment and activation of Fas-associated death domain protein, which promotes transmission of an apoptotic signal and induces apoptosis via both the extrinsic and intrinsic mitochondrial pathways $[6,8$, 9]. However, evidence indicates that cancer cells can develop resistance to TRAIL if they lose the ability to express death receptors or exhibit constant activation of anti-apoptotic pathways $[9,10]$. Certain malignant 
cells are resistant to TRAIL, including bladder cancer cells [11-13]. Therefore, identifying effective agents capable of overcoming TRAIL resistance in bladder cancer cells is necessary.

Andrographolide (Andro) is a diterpene lactone $\left(\mathrm{C}_{20} \mathrm{H}_{30} \mathrm{O}\right)$ and a major component of Andrographis paniculata Nees, which was used as a traditional anti-inflammation medicine in Asia [14, 15]. Andro can be excreted from the body over a short period of time while exhibiting low levels of toxicity to normal cells [16]. Recent progress in Andro-specific research confirmed its effectiveness and specificity in promoting cancer-cell apoptosis [17]. In this study, we discovered that Andro markedly improved the sensitivity of BLCA cells to TRAIL-induced apoptosis, and that combined treatment stimulated caspasedependent apoptosis through p53 dependent enhancement of DR4 and DR5 expression. Additionally, Andro treatment attenuated mRNA expression of $B$ cell lymphoma 2 (Bcl-2), cellular inhibitor of apoptosis 2 (cIAP2), X-linked inhibitor of apoptosis (XIAP), and nuclear factor kappa-B (NF-kB) p65 subunit (RelA). Our study provides evidence supporting clinical application of the combination therapy of TRAIL and Andrographolide for bladder cancer patients.

\section{Results}

\section{T24 cells exhibit TRAIL resistance}

As DR4 and DR5 were canonical TRAIL receptors involved in its antitumor effects, we analyzed mRNA-expression data from the Oncomine database, finding that mean DR4 mRNA expression in bladder cancer tissues exceeded that in normal bladder tissue, whereas no significant difference was found in DR5 between tumor and normal tissues (Figure 1A). Furthermore, gene set enrichment analysis (GSEA) analysis of The Cancer Genome Atlas (TCGA) profiles from BLCA patients $(n=414)$ revealed enrichment of apoptosis pathways associated with samples exhibiting high levels of both DR4 and DR5 expression (Figure 1B). Therefore, bioinformatics analysis suggested that relatively high DR4/5 expression might represent an effective therapeutic TRAIL-related target in bladder cancer cells. However, MTS assays revealed that the 50\% inhibitory concentration $\left(\mathrm{IC}_{50}\right)$ value of TRAIL was $38.35 \mathrm{ng} / \mathrm{mL}$, indicating that low concentrations of TRAIL would be ineffective in T24 cells (Figure 1C). This suggested the necessity to identify appropriate TRAIL-specific sensitizers capable of overcoming TRAIL resistance in bladder cancer cells. Moreover, Andro represents a potential agonist for TRAIL therapy, with MTS assays revealing an $\mathrm{IC}_{50}$ value for Andro of $101.5 \mu \mathrm{M}$ in T24 cells (Figure 1E).
Andro synergistically enhances TRAIL-induced inhibition of proliferation, colony formation and migration in T24 bladder cancer cells

Both cell-counting and MTS assays suggested that single treatment with either TRAIL or Andro inhibited cell-proliferation rates. Interestingly, we found that combination treatment with TRAIL and Andro substantially enhanced this inhibitory effect on cell proliferation (Figure 2A and B). Additionally, morphological changes in TRAIL and/or Androtreated cells confirmed the inhibition of T24-cell proliferation associated with combined treatment versus single treatment (Figure 2C). Moreover, colony formation dramatically decreased following combined treatment relative to that observed following treatment with Andro or TRAIL alone (Figure 2D).

Given that cancer cells exhibit potent migratory features, we conducted wound-healing assays as functional readings. The results indicated that treatment with TRAIL or Andro alone modestly decreased the ratio of migrating bladder cancer cells. In the TRAIL-treated group, the cell-migration ratio was $65.37 \pm 2.47 \%$, whereas that in the Andro-treated group was $79.65 \pm 1.82 \%$. However, combined treatment resulted in a migration ratio of $32.16 \pm$ $1.59 \%$ (Figure 2E). Evidence shows that matrix metalloproteinases (MMPs) play important roles in tumor progression, invasion, and metastasis [18]. Therefore, we evaluated protein levels of CD147 and MMP-9 by immunoblot, revealing that CD147 and MMP-9 were downregulated after a 24-h incubation with both TRAIL and Andro relative to levels observed following single treatment with TRAIL or Andro alone (Figure 2F). These findings demonstrated that combination treatment with TRAIL and Andro potently suppressed T24-cell growth and migration.

\section{Andro enhances TRAIL-induced apoptosis by initiating caspase activation in BLCA cells}

The canonical pathway associated with TRAIL-induced cell death involves binding to specific death receptors (DR4 or DR5) to initiate activation of extrinsic apoptosis [6, 7]. MTS assays suggested that in the combination-treatment groups, cell viability was further attenuated along with increasing Andro concentrations (Figure 3A). Immunoblot assays analyzing changes in protein content in T24 cells treated with TRAIL and/or Andro suggested that combined treatment enhanced caspase-dependent apoptosis based on levels of cleaved-poly (ADP ribose) polymerase 1 (PARP1; 119/89 kDa) and activation of caspases 3,9 , and 8 (Figure 3B). We then evaluated the roles of Andro in apoptosis progression 
using Annexin V-fluorescein isothiocyanate (FITC)/ propidium iodide (PI)-labeled flow cytometry. Compared with groups treated with TRAIL or Andro alone, combination treatment notably enhanced apoptosis rates in T24 cells (Figure 3C). These results showed that combined treatment of Andro and TRAIL promoted apoptosis in BLCA cells.

Additionally, we found that T24 apoptosis induced by combined treatment was initiated by caspase-specific activation that did not involve cell necrosis. T24 cell viability in the combined-treatment group was restored in the presence of the pan-caspase inhibitor Z-VAD-FMK $(57.54 \pm 4.99 \%$ for combined treatment without Z-VAD-FMK and $94.03 \pm 3.77 \%$ for combined treatment with Z-VAD-FMK). By contrast, administration of Necrostatin-1 (Nec-1; a cell-necrosis inhibitor) did not alter the decreased $\mathrm{T} 24$ cell viability observed following combined treatment with Andro and TRAIL, with cell-morphological images supporting MTS assay results (Figure 3D).

\section{Andro sensitizes TRAIL-induced apoptosis via up-regulating death receptors}

Immunoblot assays identified that Andro treatment upregulated protein levels of both DR4 and

A

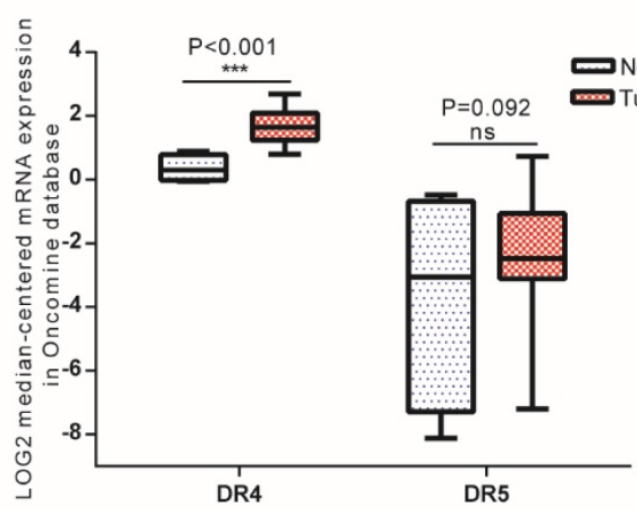

C

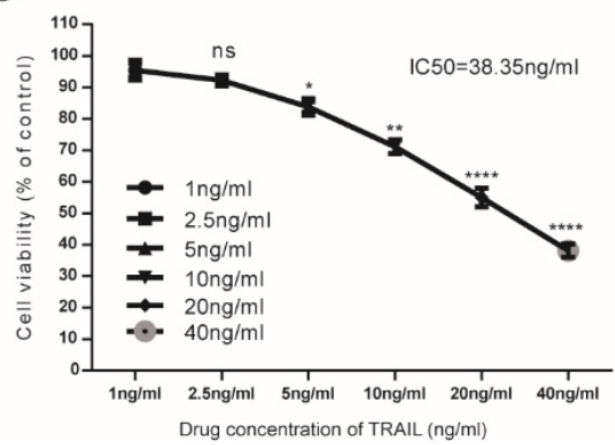

DR5 (Figure 4A). To determine whether one or both receptors were responsible for the pro-apoptotic effect of TRAIL in T24 cells, we used small-interfering (si)RNAs to block innate DR4/DR5 translation (si1201 and si955 targeting DR4 and DR5 mRNA, respectively) according to their knockdown efficiency determined by quantitative reverse transcription polymerase chain reaction (qRT-PCR) assays (Figure 4B). Following results demonstrated that cell viability was slightly restored in DR4-knockdown cells and restored to a higher degree in DR5-knockdown cells following combination treatment with TRAIL and Andro. Additionally, we observed no significant difference in cell viability between DR4- and DR5-knockdown cells (Figure 4C). Flow cytometry confirmed those from MTS assays showing that blocking DR5 translation was more effective at reducing the apoptosis rate as compared with blocking DR4 translation (apoptosis rates: $14.41 \pm$ $0.21 \%$ in DR4-knockdown cells and $8.07 \pm 0.22 \%$ in DR5-knockdown cells) (Figure 4D).

B
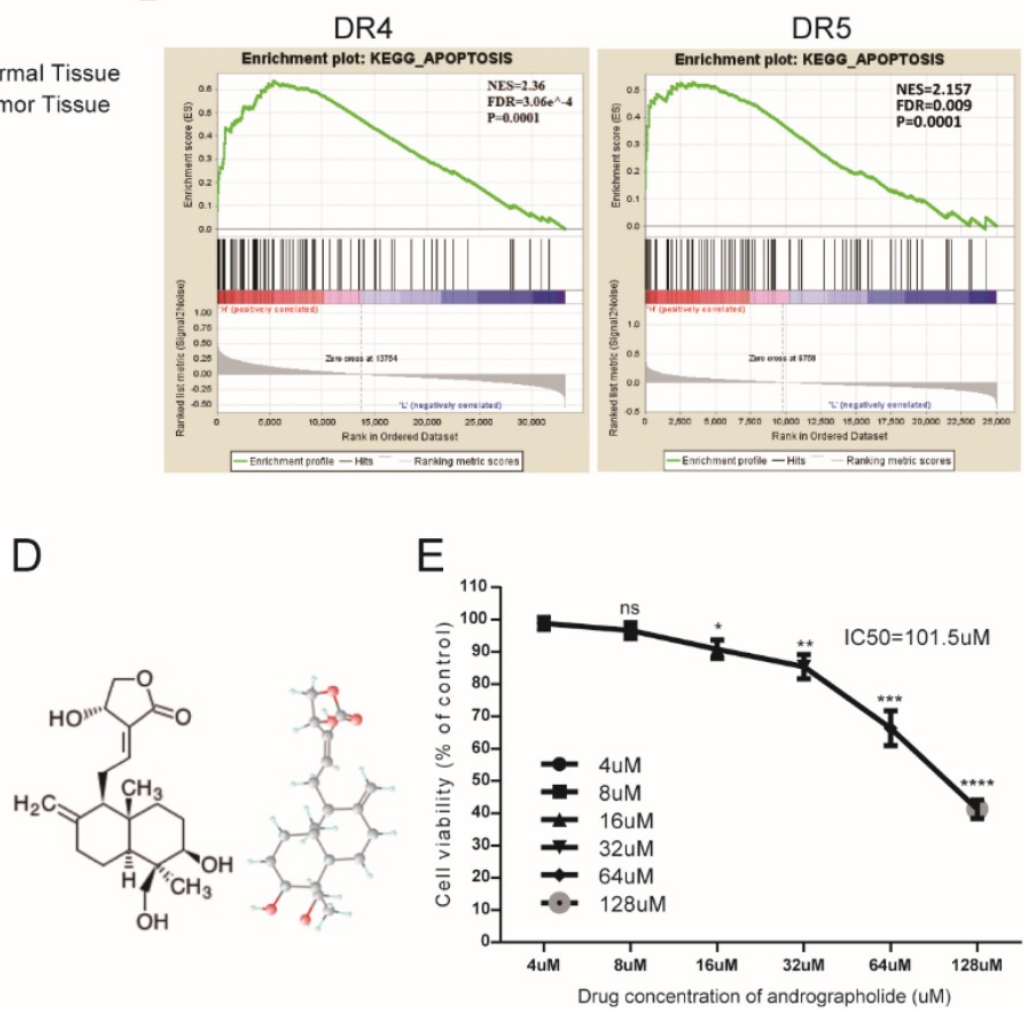

Figure 1. Potential TRAIL-receptor mRNA expression in bladder cancer patients and the antitumor effects of TRAIL and Andro in T24 cells. (A) Log2-converted DR4/DR5 mRNA expression levels from the Oncomine database. (B) GSEA results showing that high DR4/DR5 expression was positively correlated with apoptosis-gene signatures. (C) T24 cells were treated with various concentrations of TRAIL for 24-h. (D) Two- and three-dimensional chemical representation of Andro derived from the PubChem Compound Database (https://pubchem.ncbi.nlm.nih.gov/). Red, grey, and light-blue nodes represent oxygen atoms, carbon atoms, and hydrogen atoms, respectively. (E) T24 cells were treated with various concentrations of Andro for $24-\mathrm{h}$. The p-value and IC 50 values were calculated using GraphPad Prism software. Data represent the mean $\pm \mathrm{SD}$. $* \mathrm{P}<0.05 ; * * \mathrm{P}<0.01 ; * * * \mathrm{P}<0.001(n=3)$. 

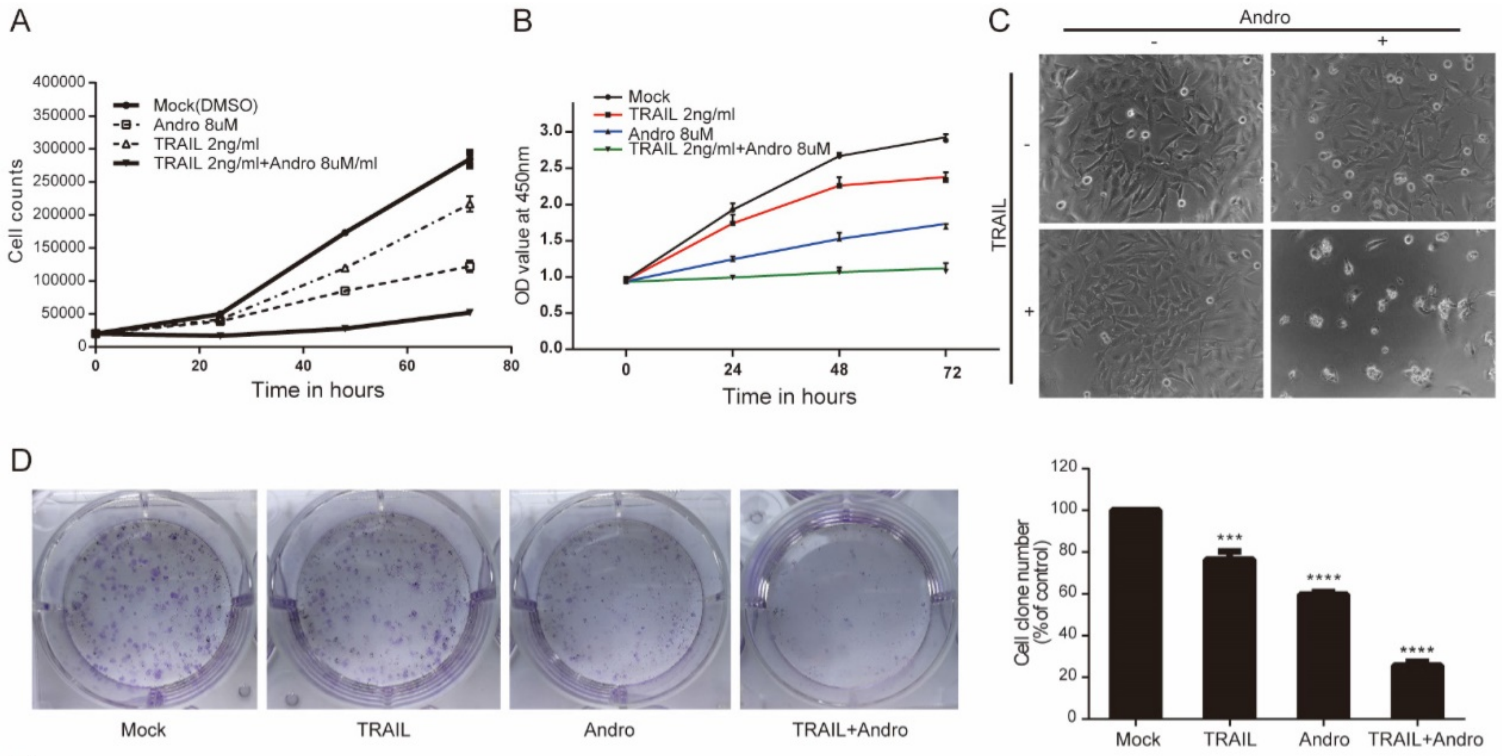

$\mathrm{E}$
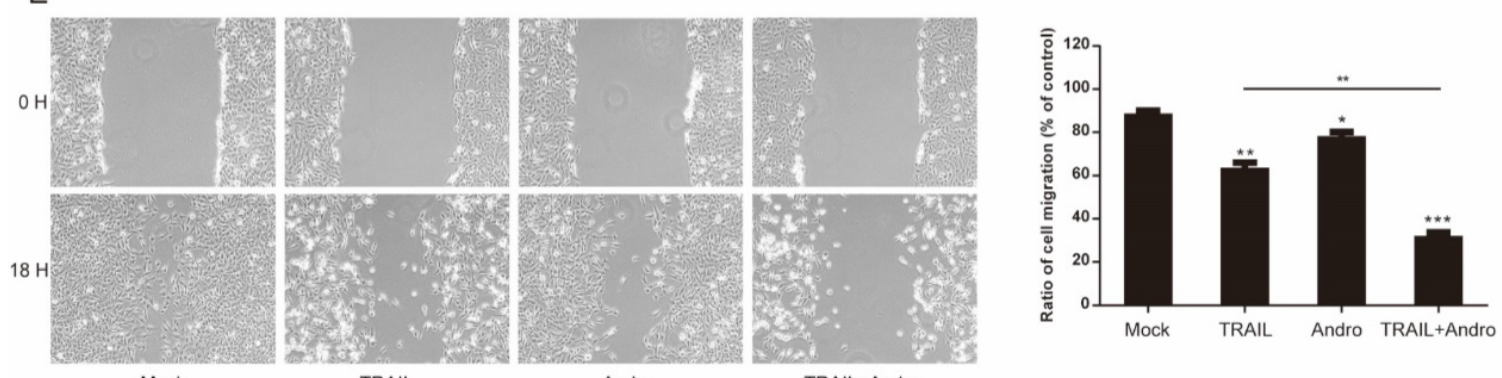

$\mathrm{F}$
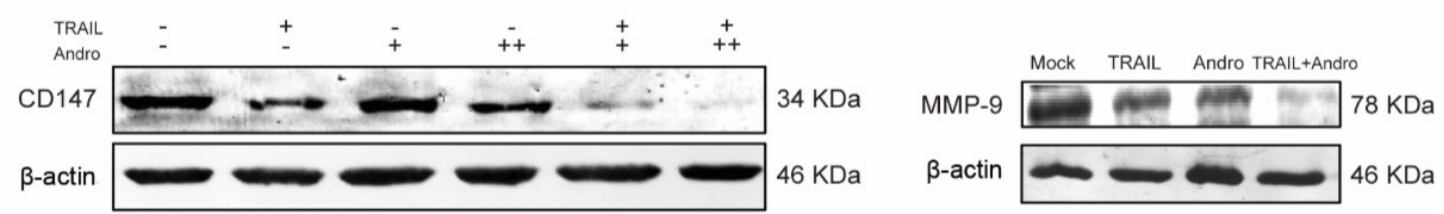

Figure 2. TRAIL combined with Andro further inhibits T24-cell proliferation, migration, and colony formation. (A, B) Effects of TRAIL and/or Andro treatment on the T24 growth curve. Verification by cell-counting and MTS assays. (C) Images (200x) show T24 cells following treatment with TRAIL or/and Andro for 72-h. (D) Effects of TRAIL and Andro treatment on the colony formation of BLCA cell lines. T24 cells were treated with DMSO (control), TRAIL ( $2 \mathrm{ng} / \mathrm{mL})$, or Andro ( $8 \mu \mathrm{M})$ alone or both TRAIL $(2 \mathrm{ng} / \mathrm{mL})$ and Andro $(8 \mu \mathrm{M})$ and incubated for 12 days. Cell colonies ( $>50$ cells) were counted using an inverted microscope (100x). (E) Effects of TRAIL and Andro treatment on T24-cell migration. T24 cells were treated with DMSO, TRAIL $(2 \mathrm{ng} / \mathrm{mL})$, and/or Andro $(5 \mu \mathrm{M})$ for $18 \mathrm{~h}$. Images (100x) show T24-cell migration after treatment. (F) Left panel: the protein levels of CD147. Right panel: MMP-9 in T24 cells treated with different concentrations of TRAIL (2 ng/ml) and/or Andro [4uM (+) or 8 uM (++)] for 18-h and measured by western blot. Data represent the mean \pm SD. $* P<0.05 ; * * P<0.01 ; * * * P<0.001(n=3)$.

\section{p53 is required for Andro-induced DR4/DR5 upregulation and enhancement of cytotoxicity}

Because combined treatment enhanced DR4 and DR5 protein levels in T24 cells (Figure 4A), we performed GSEA of the TCGA profiles of 414 bladder patients, which indicated that both high TNFRSF10A and TNFRSF10B expression were positively correlated with the $p 53$-signaling pathway (Figure 5A). Therefore, we constructed a p53-knockdown T24 cell line to explore the molecular mechanisms associated with Andro-mediated TNFRSF10B expression. Following qRT-PCR and immunoblot confirmation of p53-knockdown efficiency (Figure 5B), immunoblot results revealed that Andro enhanced $p 53$ levels while p53 knockdown attenuated Andro-induced upregulation of DR4 and DR5 levels in T24 cells
(Figure 5C). Moreover, MTS assays demonstrated partial restoration of cell viability in p53-knockdown cells compared to sh-ctrl T24 cells following combined treatment with TRAIL and Andro (control shRNA, $69.80 \pm 4.89 \%$ vs. $p 53$ shRNA, $84.32 \pm 2.17 \%$ ) (Figure $5 \mathrm{D}$ ). Knocking down of $p 53$ expression also contributed to attenuated synergistic effect of Andro for which decreased cell apoptosis rate in TRAIL-treated T24 cells (control shRNA, $23.88 \pm 0.77 \%$ vs. p53 shRNA, $13.99 \pm 0.88 \%$ ) (Figure S1).

\section{Andro interrupts NF-KB pathway further sensitizes BLCA cells to TRAIL-induced apoptosis}

Here, we found that RelA (the NF-kB p65 subunit) was downregulated at both the mRNA and 
protein levels following combined treatment with TRAIL and Andro (Figure 6A-C). Additionally, combined treatment modified protein and mRNA levels of apoptosis-related genes, resulting in decreased cIAP2 protein levels (Figure 6A), Bcl-2 mRNA levels (combined treatment, $63.08 \pm 1.54 \%$ vs. TRAIL or Andro treatment alone, $104.33 \pm 18.3 \%$ and $90.76 \pm 1.77 \%$, respectively), and attenuated XIAP mRNA levels (which were stimulated by TRAIL treatment alone) following Andro treatment (Figure $6 \mathrm{C})$. To confirm the role of the NF- $\mathrm{BB}$ pathway in
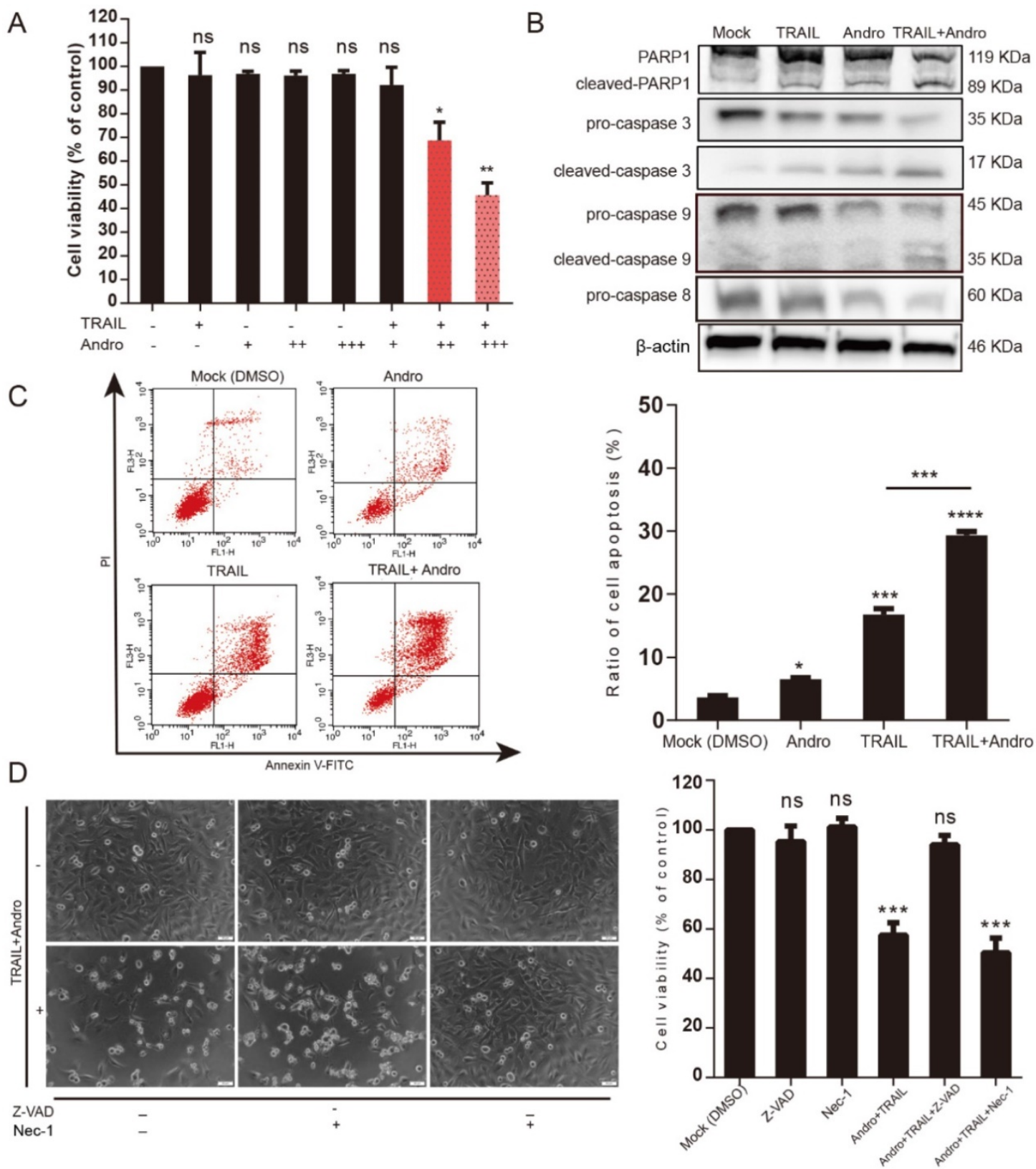

Figure 3. Andro sensitizes TRAIL-induced apoptosis but not necrosis in T24 cells. (A) TRAIL combined with Andro treatment inhibited the cell viability of BLCA cell lines. T24 cells were treated with various concentrations of TRAIL and/or Andro [2 (+) or $4(++)$ or $8(+++) \mu M]$ for $24-h$, and cell viability was examined by MTS assay. (B) Protein expression of hallmarks of apoptosis in T24 cells treated with $2 \mathrm{ng} / \mathrm{mL}$ TRAlL or/and $8 \mu \mathrm{M}$ of Andro for 24-h. (C) Effects of TRAlL and Andro treatment on apoptosis in T24 cell lines. Cells were identified by Annexin V-FITC detection following DMSO (control), TRAIL ( $2 \mathrm{ng} / \mathrm{mL})$, and/or Andro $(8 \mu M)$ treatment for $24-h$. Histogram showing the apoptosis rates of all cell lines. (D) Images $(200 \times)$ showing apoptotic cells after treatment under different conditions. Histograms of T24 cells treated with DMSO, TRAIL (2 $\mathrm{ng} / \mathrm{mL})$, and/or Andro $(8 \mu \mathrm{M})$ and Z-AVD-FMK $(0.05 \mu \mathrm{M})$ or Nec-1 $(0.05 \mu \mathrm{M})$. Cell viability was determined by MTS assay after $24-\mathrm{h}$. Data represent the mean \pm SD. $* \mathrm{P}<0.05$; **P $<0.01$; *** $\mathrm{P}<0.001(n=3)$. 
A

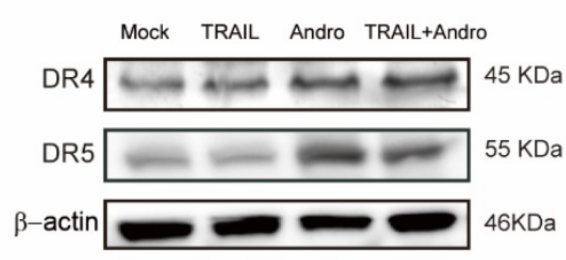

C

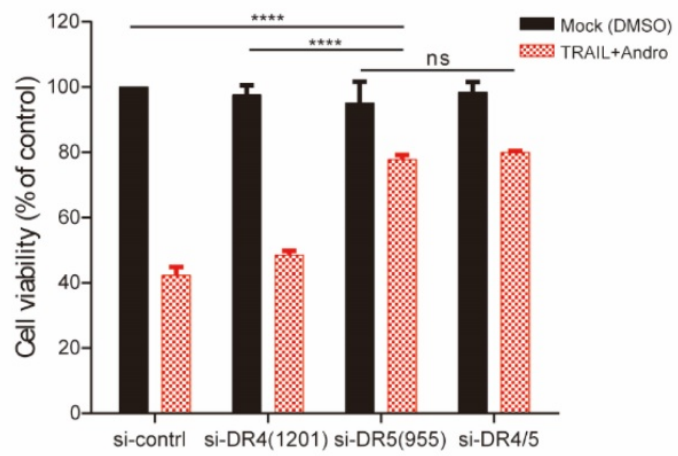

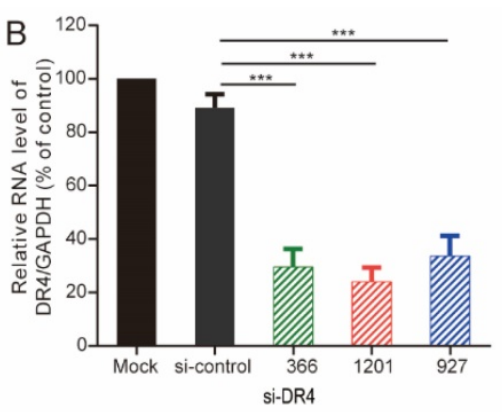

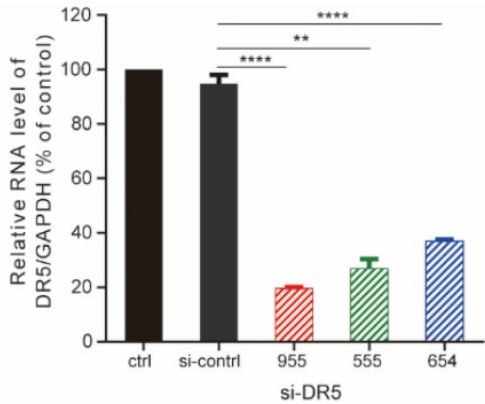

D

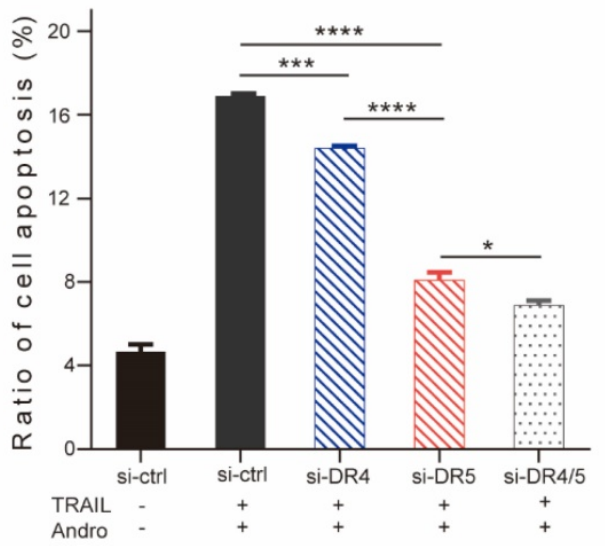

Figure 4. DR5 is critical for Andro-mediated sensitization of T24 cells to TRAIL. (A) DR4/DR5 protein levels in T24 cells treated with TRAIL and/or Andro for 24-h. (B) Histograms of qRT-PCR screening for DR4 (left panel)/DR5 (right panel) siRNA optimization. (C) Cell viability of T24 cells transfected with either siDR4 or siDR5 or both and treated with a combination of TRAIL and Andro for 24-h. (D) Effects of TRAIL and Andro combination treatment on apoptosis in DR4-knockdown and/or DR5-knockdown T24 cell lines. Histogram showing the apoptosis rates of all cell lines. Data represent the mean \pm SD. $* \mathrm{P}<0.05 ; * * \mathrm{P}<0.01 ; * * * \mathrm{P}<0.001(n=3)$.

A

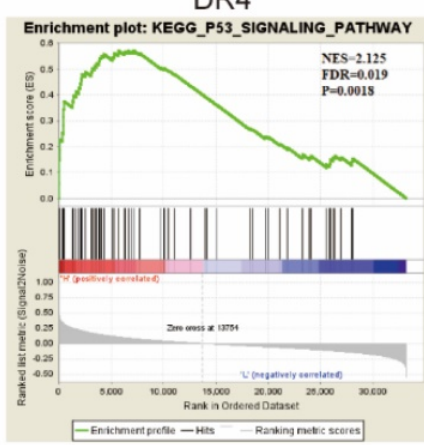

C

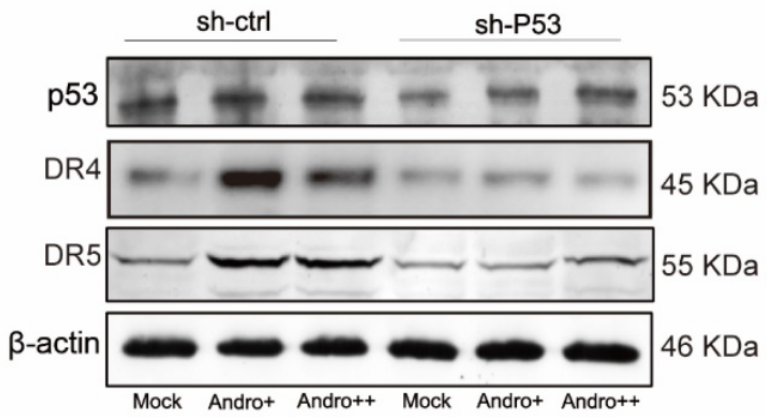

B
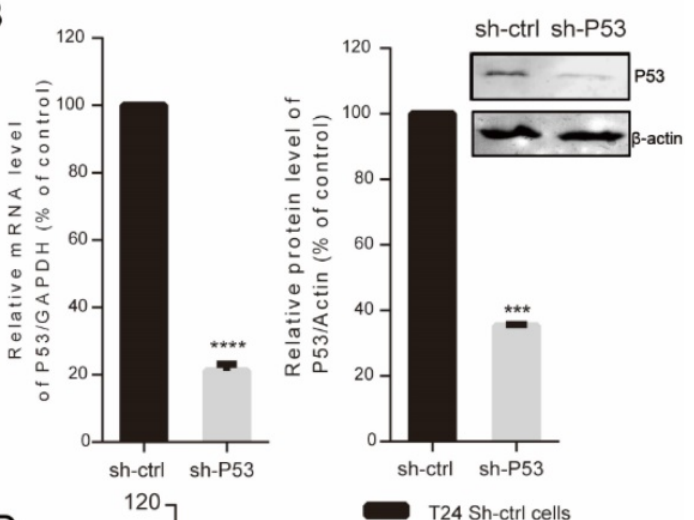

D

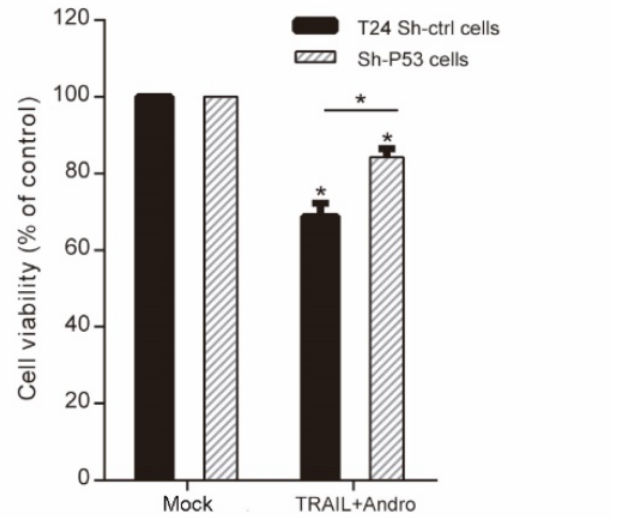

Figure 5. Activation of $\mathbf{p 5 3}$ expression is required for Andro-induced upregulation of DR4/DR5. (A) GSEA revealing that genes in the high DR4/DR5-expression group were positively correlated with signatures related to the p53-signaling pathway. (B) p 53 mRNA (left panel) and p53 protein levels (right panel) in control shRNA- and $p 53$ shRNA-transfected T24 cells. (C) DR4/DR5 protein levels in control shRNA and p53 shRNA-transfected cells treated with Andro [4 (+) or $8 \mu M(++)$ ]. (D) Cell viability of T24 cells transfected with control shRNA or $\mathrm{p} 53$ shRNA and treated with a combination of TRAlL $(2 \mathrm{ng} / \mathrm{mL})$ and Andro $(8 \mu M)$ for $24-\mathrm{h}$. Data represent the mean \pm SD. *P $<0.05$; $* * \mathrm{P}<0.01$; *** $\mathrm{P}<0.001(n=3)$. 
A

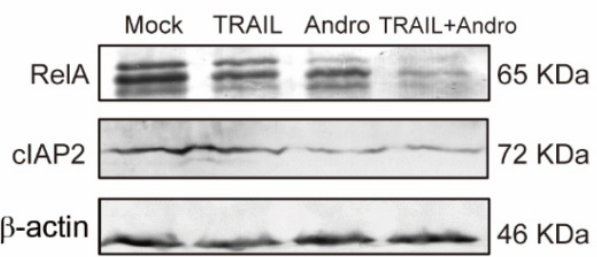

B

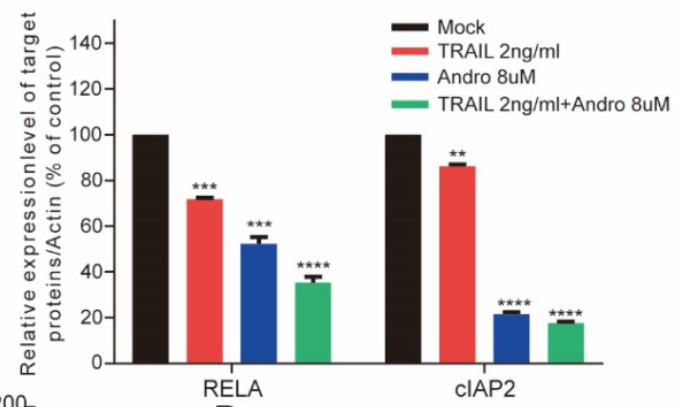

C

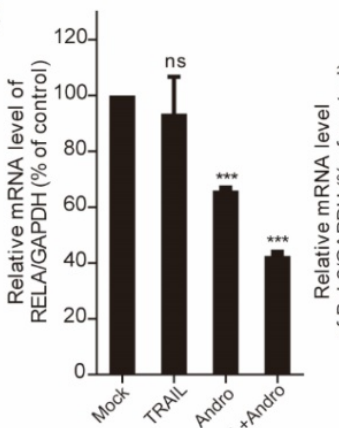

E

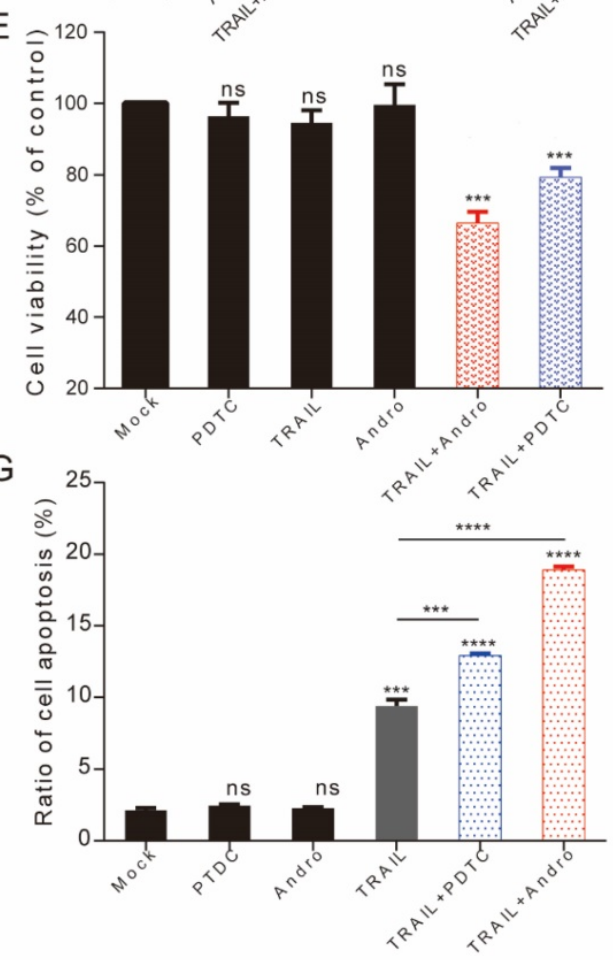

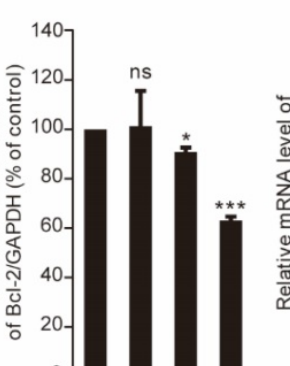

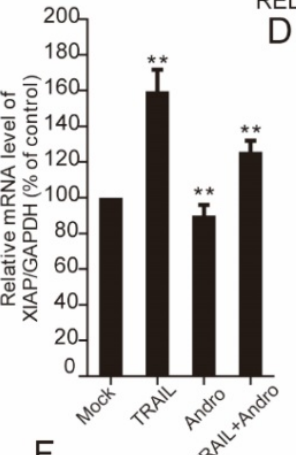

D 120
$\mathrm{F}$
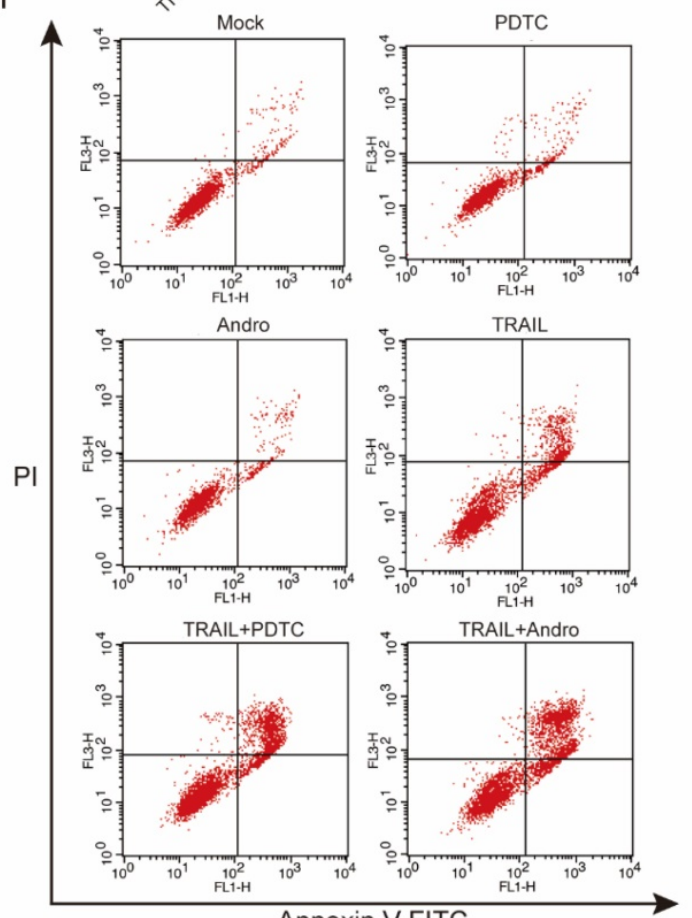

Annexin V-FITC

Figure 6. Combination treatment with TRAIL and Andro enhances cell apoptosis rates through inhibiting the NF-KB pathway in T24 cells. (A, B) T24 cells were treated with $2 \mathrm{ng} / \mathrm{mL}$ TRAIL and/or $8 \mu \mathrm{M}$ Andro for 24-h, RelA/clAP2 protein levels and its quantification were assessed by immunoblot. (C) Histograms showing the relative mRNA levels of RelA, Bcl-2, and XIAP in T24 cells treated with TRAlL or/and Andro. (D) T24 cells were treated with PDTC at various concentrations for 24-h, and cell viability and $\mathrm{IC}_{50}$ values were measured using MTS assays. (E) T2 cells were treated with $2 \mathrm{ng} / \mathrm{mL}$ TRAIL or $5 \mu M$ PDTC or $8 \mu M$ Andro or with a combination of TRAIL and Andro/PDTC for 24-h. Cell viability was measured by MTS assay. (F, G) Cell apoptosis rates of T24 cells according to Annexin V-FITC detection. T24 cells were treated with 2 $\mathrm{ng} / \mathrm{mL}$ TRAIL or $5 \mu \mathrm{M}$ PDTC or $8 \mu \mathrm{M}$ Andro or with a combination of TRAIL and Andro/PDTC for 24-h. Histograms showing the apoptosis rates. Data represent the mean \pm SD. $* \mathrm{P}<0.05$; **P $<0.01$; ***P $<0.001(n=3)$.

\section{The synergistic effect of Andro on TRAIL is effective in multiple BLCA cell lines}

We performed MTS assays to evaluate the effect of TRAIL and/or Andro treatment on 5637 and UM-UC 3 bladder cancer cell lines. We found that the $\mathrm{IC}_{50}$ value for TRAIL and Andro treatment alone of 5637 cells was $15.19 \mathrm{ng} / \mathrm{mL}$ and $82.9 \mu \mathrm{M}$, respectively
(Figure 7A), whereas that for UM-UC 3 cells was 75.00 $\mathrm{ng} / \mathrm{mL}$ and $103.3 \mu \mathrm{M}$, respectively (Figure $7 \mathrm{C}$ ). Both UM-UC 3 cells and 5637 cells were insensitive to low concentrations of either TRAIL or Andro, whereas combined treatment displayed a clear inhibitory effect on the cell viability of these TRAIL-resistant BLCA cell lines (Figure 7B and D). At 24-h post-treatment of 
UM-UC 3 cells with both Andro and TRAIL, cell viability was $71.54 \pm 5.06 \%$ as compared with $92.65 \pm$ $3.43 \%$ following treatment with TRAIL alone. Similarly, in 5637 cells, cell viability following combined treatment was $57.39 \pm 8.31 \%$ as compared with $85.96 \pm 3.76 \%$ following TRAIL treatment alone. Additionally, cell apoptosis rates determined by FACS revealed that combination treatment enhanced apoptosis in both 5637 and UM-UC 3 cells (Figure 7E). Moreover, the synergistic effect of PDTC on TRAIL induced apoptosis was also validated in both 5637 and UM-UC 3 BLCA cell lines (Figure 8). These results further supported that Andro sensitized multiple BLCA cell lines to TRAIL-mediated apoptosis by inhibiting the NF- $\mathrm{kB}$ signaling pathway.
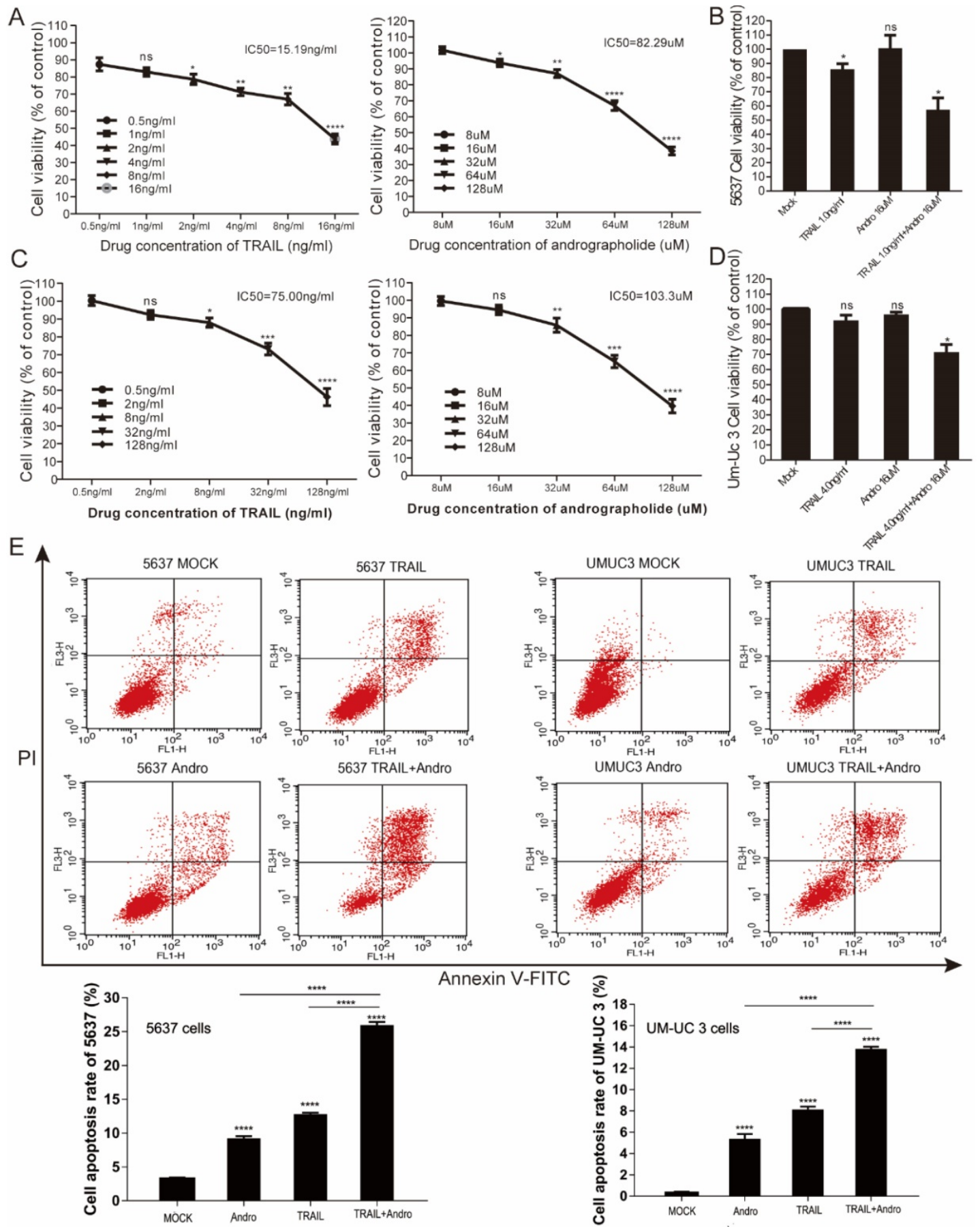

Figure 7. Andro enhances TRAIL-mediated suppression of cell viability and apoptosis rates in other BLCA cell lines. (A, B) 5637 cells were treated with various concentrations of TRAIL or/and Andro for 24-h, and cell viability and IC 50 values were measured by MTS assays. (C, D) UM-UC 3 cells were treated with various concentrations of TRAIL or/and Andro for 24-h, and cell viability and IC 50 values were measured by MTS assays. (E) Effects of TRAIL and Andro treatment on 5637 and UM-UC 3 cell apoptosis. 5637 cells were identified by Annexin V-FITC detection following DMSO (control), TRAIL $(2 \mathrm{ng} / \mathrm{mL})$, or Andro (10 $\mu M)$ treatment and combined TRAIL ( $2 \mathrm{ng} / \mathrm{mL})$ and Andro $(10 \mu \mathrm{M})$ treatment for $24-\mathrm{h}$. UM-UC 3 cells were identified by Annexin V-FITC detection following DMSO (control), TRAIL (10 ng/mL), or Andro (20 $\mu$ M) treatment and combined TRAIL $(10 \mathrm{ng} / \mathrm{mL})$ with Andro $(20 \mu \mathrm{M})$ treatment for $24-\mathrm{h}$. Histogram showing the apoptosis rates of 5637 and UM-UC3 cell lines. Data represent the mean \pm SD. *P $<0.05$; **P $<0.01 ; * * * \mathrm{P}<0.001(n=3)$. 
A
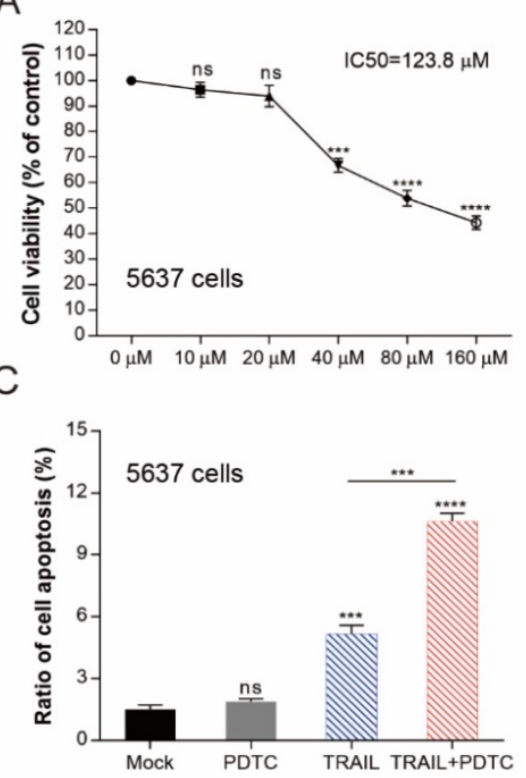

D
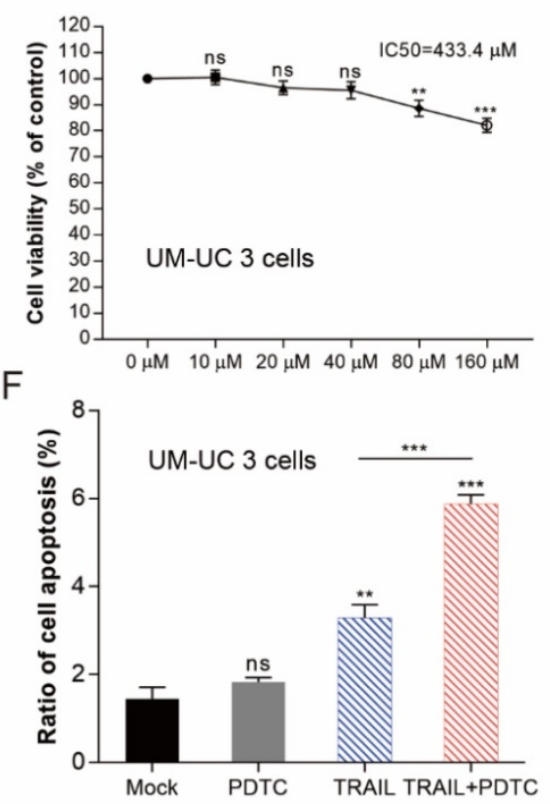

B
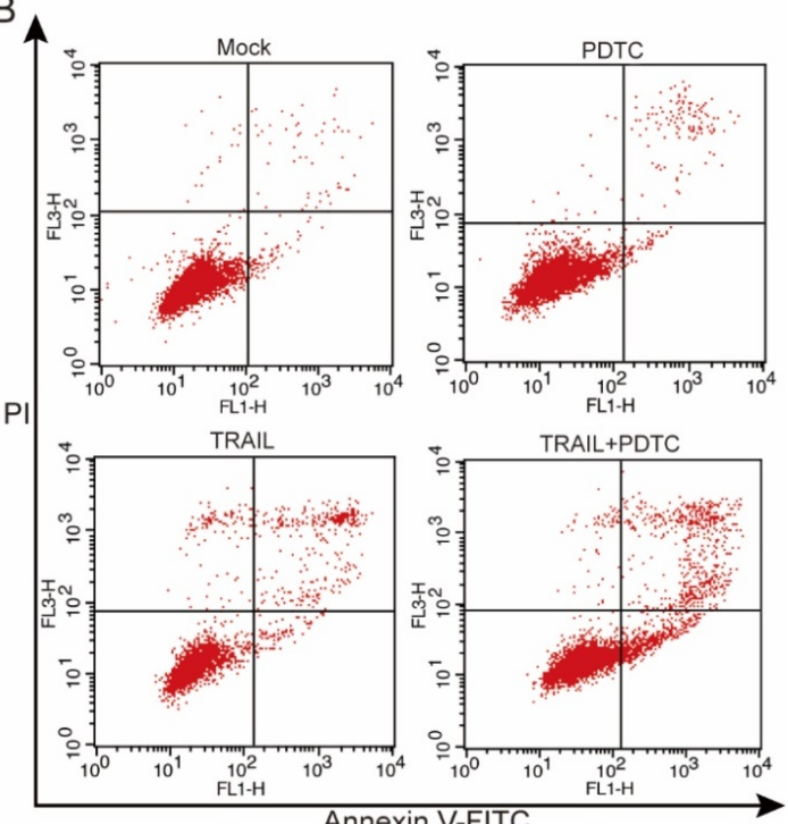

Annexin V-FITC

E
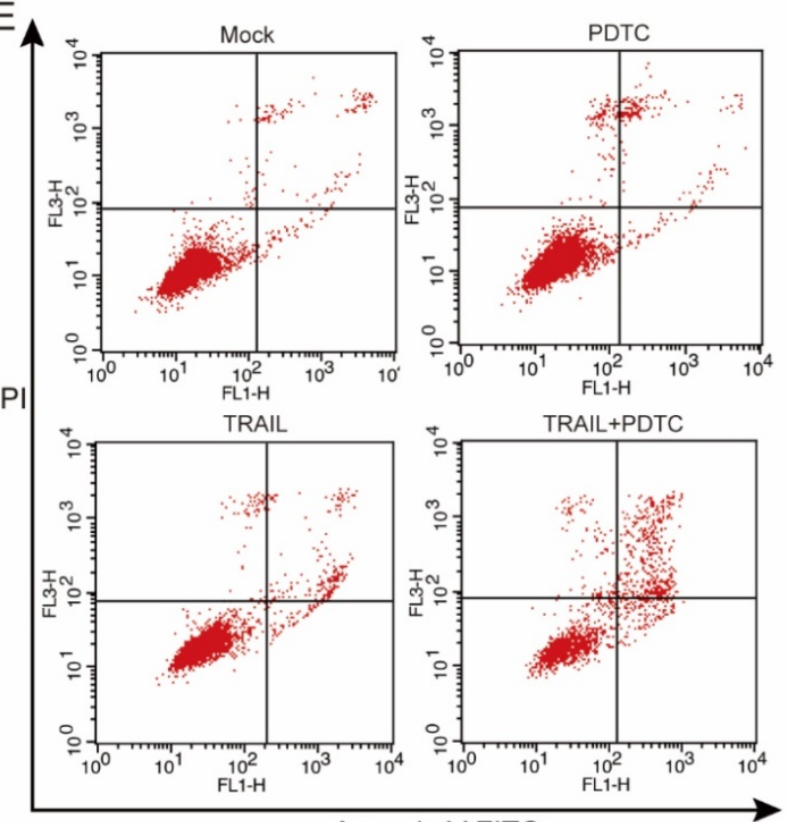

Figure 8. Enhancing TRAIL induced apoptosis through blocking NF- $\mathrm{KB}$ pathway is effective in multiple BLCA cell lines. (A) 5637 cells were treated with various concentrations of PDTC for 24-h, cell viability and IC 50 values were measured by MTS assays. (B) Effects of TRAIL and PDTC treatment on 5637 cell apoptosis. 5637 cells were identified by Annexin V-FITC detection after DMSO (Mock), TRAIL $(2 \mathrm{ng} / \mathrm{mL})$, or PDTC $(20 \mu \mathrm{M})$ treatment and combined TRAIL $(2 \mathrm{ng} / \mathrm{mL})$ and PDTC $(20 \mu \mathrm{M})$ treatment for 24-h. (C) Histogram showing the apoptosis rates of 5637 cells. (D) UM-UC 3 cells were treated with various concentrations of PDTC for $24-h$, cell viability and IC 50 values were measured by MTS assays. (E) Effects of TRAIL and PDTC treatment on UM-UC 3 cell apoptosis. UM-UC 3 cells were identified by Annexin V-FITC detection after DMSO (control), TRAIL $(10 \mathrm{ng} / \mathrm{mL})$, or PDTC $(40 \mu \mathrm{M})$ treatment and combined TRAIL $(10 \mathrm{ng} / \mathrm{mL})$ with PDTC (40 $\mu \mathrm{M})$ treatment for $24-\mathrm{h}$. (F) Histogram showing the apoptosis rates of UM-UC 3 cells. Data represent the mean \pm SD. $* P<0.05$; **P $<0.01$; ***P $<0.001(n=3)$.

\section{Discussion}

The unique property of TRAIL triggering both extrinsic and intrinsic apoptosis making it a potentially effective anticancer agent $[4,19,20]$. Resistance of cancer cells to TRAIL-mediated apoptosis represents a major limitation to its clinical application [21]. Therefore, identifying agents capable of enhancing TRAIL-induced anticancer effects is necessary. In the present study, clinical database analysis and GSEA revealed that mRNA expression of death receptors of TRAIL is positively associated with apoptosis signaling pathway in BLCA patients (Figure 1A and B). However, previous studies reported that human BLCA cells were resistant to TRAIL treatment [22]. Thus, our findings that Andro, a natural compound, enhanced the sensitivity of 
BLCA cell lines to TRAIL-mediated apoptosis provided critical insight into a novel therapeutic strategy for BLCA patients.

In this study, we confirmed the synergetic effect of combined Andro and TRAIL treatment in bladder cancer cells, with Andro treatment enhancing TRAIL-mediated inhibition of cell viability, proliferation, migration, and colony formation and promotion of apoptosis in T24 cells. Consistently, we found that protein levels of two factors involved in tumor migration (MMP-9 and CD147) [23, 24] were decreased following combination treatment (Figure 2). A pan-caspase inhibitor (Z-VAD-FMK), almost completely restored cell viability of T24 cells treated with combination therapy, further supporting that Andro-specific increased TRAIL mediated caspasedependent apoptosis in T24 cells (Figure 3). Another important finding was that combination therapy also increased TRAIL-induced apoptosis rates in multiple BLCA cell lines, indicating that Andro enhanced the anticancer efficacy of TRAIL independent of BLCA cell type (Figure 7).

TRAIL binding to its death receptors (DR4 and DR5), it initiates the formation of the DISC complex, which leads to activation of caspase cascades and induction of apoptosis [25]. However, in cancer cells, pro-survival signaling pathways, including the NF-kB pathway, are activated to prevent TRAIL-induced

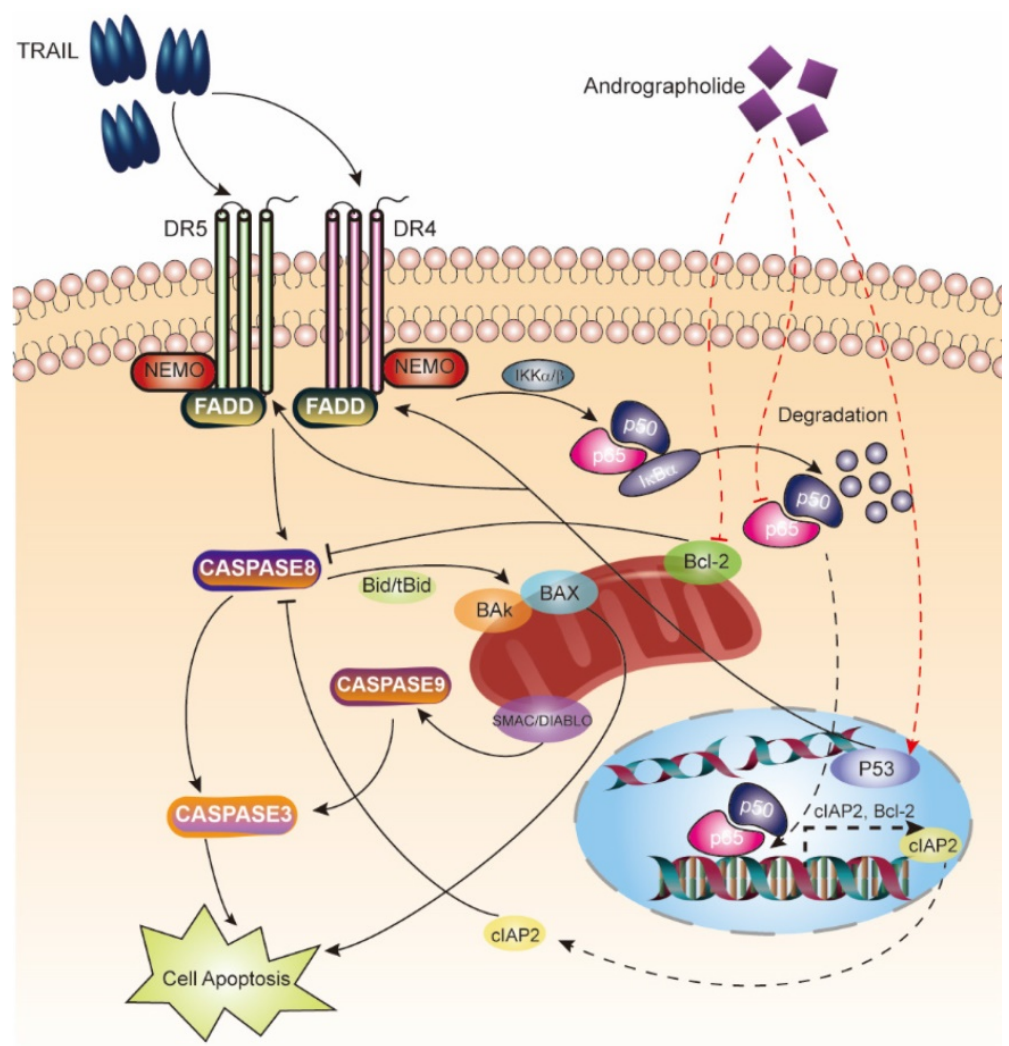

Figure 9. Schematic map describing apoptosis induction of the TRAIL and Andro combination therapy. apoptosis, with accumulating evidence supporting elevated NF-KB levels observed in bladder cancers $[26,27]$. Two strategies should be considered to enhance the therapeutic efficacy of TRAIL: 1) enhancing the activation of apoptosis pathways and 2) suppressing pro-survival pathways.

We noted that T24 cells treated with Andro exhibited substantial increases in both DR4 and DR5 levels, which possibly contributed to TRAIL-mediated apoptosis. It remains controversial whether DR4 or DR5 represents the determinant receptor associated with TRAIL-induced apoptosis. Previous studies claimed that DR4 was more effective in promoting TRAIL-mediated cancer-cell apoptosis [28-30]; whereas other studies indicated DR5 was critical for promoting TRAIL-induced cancer-cell apoptosis [25]. Here, by using siRNA targeting DR4 or DR5, we confirmed that both DR4 and DR5 were required for tumor suppressing activity by combined treatment with Andro and TRAIL in BLCA cells. Crucially, we noticed that silencing DR5 in BLCA cells is more effective in restoring the resistance for Andro/TRAIL treatment than silencing DR4, indicating that DR5 expression level is the major determinant for the sensitization by Andro in BLCA cells. Hence, we suggested that upregulated DR5 rather than DR4 enhanced TRAIL-induced apoptosis in T24 cells (Figure 4).

Our bioinformatics analysis of BLCA patients' data revealed a correlation between death receptors of TRAIL and the p53-signaling pathway. In consistent with previous studies [29], we confirmed that p53 expression is critical for Andromediated increases in DR4 and DR5 expression (Figure 5). Downregulation of endogenous p53 expression blocked Andromediated upregulation of DR4 and DR5 levels in T24 cells. Importantly, decreased p53 partially attenuated the synergistic antitumor effects of the combination treatment. Therefore, we assumed that other pathways were involved in cancer-cell apoptosis associated with combined Andro and TRAIL treatment.

The NF-kB-signaling pathway protects tumor cells from apoptosis by upregulating multiple anti-apoptosis genes [31]. Accumulating evidence shows that activation of the NF-kB subunit RelA renders tumor cells resistant to TRAIL-mediated apoptosis [32]. In the present study, we found that combined treatment of T24 cells with Andro and TRAIL decreased RelA expression at both mRNA and protein levels. 
Additionally, the mRNA expression of several NF-кB-regulated anti-apoptosis genes, including cIAP2, Bcl-2, and XIAP, was attenuated following Andro treatment. Therefore, we hypothesized that inhibiting the NF-кB-dependent pro-survival pathway by decreasing RelA expression might sensitize T24 cells to TRAIL-induced apoptosis. To further support our conclusion, we found that using PDTC (a pan- NF- $\kappa B$ inhibitor capable of suppressing both NF-KB-DNA binding and NF-KB-dependent transcriptional activity) enhanced TRAIL-mediated apoptosis and suppression of cell viability in multiple BLCA cell lines. These results suggested that the synergistic effect of combined treatment and associated NF- $\mathrm{KB}$-pathway inhibition was correlated with the downregulation of RelA by Andro treatment (Figure 6), which agreed with previously reported results [33, 34]. As all our experiments were performed in vitro, thus it is still needed to assess the in vivo effect of the combination therapy of TRAIL and Andro on bladder cancer in future studies.

Our findings indicated that combined treatment with TRAIL and Andro increased DR4/DR5 levels and suppressed the NF- $\kappa \mathrm{B}$-dependent anti-apoptosis pathway, both of which contributed to BLCA-cell sensitization to TRAIL. In conclusion, these results indicated that Andro treatment acted synergistically with TRAIL treatment and offers a potential treatment option for patients with TRAIL-resistant bladder cancer.

\section{Materials and Methods}

\section{Microarray data processing and visualization}

RNA-seq data from the transcriptome profile in a bladder cancer dataset was downloaded from TCGA (https://portal.gdc.cancer.gov/repository; data release 10.1; February 15, 2018) and contained 414 bladder tumor tissues and 19 adjacent normal bladder tissue samples. TNFRSF10A and TNFRSF10B mRNA expression data from a previous study [35] was downloaded from the Oncomine database (https:// www.oncomine.org/resource/main.html). GSEA was performed by GSEA 3.0 (BROAD Institute; http:// www.broadinstitute.org/gsea) [36]. Data processing and visualization were accomplished by $\mathrm{R}$ (https: //www.r-project.org/) and GraphPad Prism (v7.0; GraphPad Software, La Jolla, CA, USA).

\section{Cell culture and treatments}

The BLCA cell lines T24, UM-UC 3, and 5637 cells were purchased from the Type Culture Collection of the Chinese Academy of Sciences (Shanghai, China), and cells were cultured in Dulbecco's modified Eagle medium (HyClone, Logan,
UT, USA). All media were supplemented with $10 \%$ fetal bovine serum (Biological Industries, Cromwell, CT, USA). All cells were cultured under standard incubator conditions $\left(37^{\circ} \mathrm{C}\right.$ and $\left.5 \% \mathrm{CO}_{2}\right)$.

\section{Reagents and kits}

Andrographolide was dissolved in $10 \mathrm{mM}$ dimethyl sulfoxide (DMSO) used as a stock solution, and recombinant human TRAIL (R\&D Systems, MN, USA) was prepared in phosphate-buffered saline (PBS) containing $0.1 \%$ bovine serum albumin at 20 $\mu \mathrm{g} / \mathrm{ml}$. Z-VAD-FMK (HY-16658), Necrostatin-1 (HY-15760), and PDTC (HY-18738) were purchased from MedChemExpress (Monmouth Junction, NJ, USA). Antibodies included the following: caspase-8, caspase-9, RelA, caspase-3, and DR4 from Cell Signaling Technology (Danvers, MA, USA); $\beta$-actin, PARP1, and cIAP2 from Abcam (Cambridge, UK); $p 53$ from Thermo Fisher Scientific (Waltham, MA, USA); and DR5 from Biolegend (307407; San Diego, CA, USA).

\section{Cell-proliferation assay}

We used cell-growth curves and colony formation assays to evaluate the effect of Andrographolide and/or TRAIL on bladder cancer-cell proliferation. For growth assays, $\sim 2 \times 10^{4}$ cells/well were seeded in a 24-well plate, treated with different concentrations of Andrographolide or/and TRAIL or DMSO (as control), and trypsinized, followed by resuspension and counting using a cell-counting chamber (Shanghai Precision Instruments Co., Ltd., Shanghai, China). Cell number was counted and recorded after 24-h, 48-h, and 72-h, with the optical density at $450 \mathrm{~nm}$ measured at the same time points.

For colony formation assays, $\sim 200$ cells/well were seeded in a 6-well plate with $2 \mathrm{~mL}$ medium, treated under different conditions, and incubated for 12 days, after which cells were washed with cold PBS and fixed with $4 \%$ paraformaldehyde for $20 \mathrm{~min}$. Cells were then stained with $0.1 \%$ crystal violet solution for $15 \mathrm{~min}$ at room temperature, washed with double-distilled water three times, and air dried. Colonies ( $>50$ cells) were counted using an inverted microscope $(100 \times)$. All experiments were performed in triplicate.

\section{Cell-viability assay}

Cell viability was assessed by measurement of formazan production after the addition of MTS (Promega, Madison, WI, USA). Nearly 6000 cells/well were seeded in a 96 -well plate and incubated at $37^{\circ} \mathrm{C}$ in a $5 \% \mathrm{CO}_{2}$ incubator for 24-h. Cells were then incubated in $100 \mu \mathrm{L}$ of cell-culture medium containing $20 \mu \mathrm{L}$ MTS for 2 -h at $37^{\circ} \mathrm{C}$, followed by the application 
of different treatment conditions for 24-h. Absorbance was detected using a BioTek ELISA reader (Winooski, VT, USA) at a wavelength of $450 \mathrm{~nm}$. Cell-inhibitory ratio was calculated using the following formula:

$100 \times$ cell growth inhibitory ratio $(\%)=100 \times[(\mathrm{A} \times$
450 control - A450sample $) /($ A450control - A450blank $)]$

\section{Immunoblot assay}

Cells in 6-well plates were washed twice with cold PBS. RIPA lysis buffer (Wanleibio, Beijing, China) was then added to the wells, followed by incubation for $15 \mathrm{~min}$ at $4^{\circ} \mathrm{C}$. Whole-cell lysate was centrifuged at $12,000 \mathrm{~g}$ at $4^{\circ} \mathrm{C}$, and the supernatant was incubated with loading buffer and denatured at $100^{\circ} \mathrm{C}$ for $15 \mathrm{~min}$, followed by sodium dodecyl sulfate polyacrylamide gel electrophoresis. The separated proteins were transferred to nitrocellulose membranes (Millipore, Billerica, MA, USA), and 5-bromo-4-chloro-3-indolyl phosphate and nitro blue tetrazolium (Millipore) were used to visualize the protein bands, with a Quantity One instrument (Bio-Rad, Hercules, CA, USA) used for imaging. Secondary antibodies included anti-rabbit IgG and anti-mouse IgG (Cell Signaling Technology, USA).

\section{Flow cytometric analysis}

Cells treated under different conditions for 24-h were resuspended in 6-well culture plates, washed twice with cold PBS, and pelleted by centrifugation at $5000 \mathrm{~g}$ for $5 \mathrm{~min}$. Apoptotic cells were quantified using an Annexin V-FITC apoptosis detection kit (KeyGEN BioTECH, Nanjing, China). A FACS flow cytometer (BD Biosciences, San Diego, CA, USA) was used to analyze cell apoptosis.

\section{qRT-PCR}

Briefly, RNA isolated from cells was converted to cDNA using a reverse transcription kit (Transgen Biotech, Beijing, China). PCR amplification was performed using a Roche LC480 instrument (Roche, Basel, Switzerland) with SYBR Green supermix (Fermentas, Waltham, MA, USA), $1 \mu \mathrm{M}$ of each primer, and $6 \mu \mathrm{L}$ of diluted cDNA. Samples were amplified in triplicate. Primer sequences for glyceraldehyde 3-phosphate dehydrogenase (GAPDH), Bcl-2, XIAP, RelA, and p53 are listed in Table S1. Relative gene-expression levels were represented as $\Delta \mathrm{Ct}=\mathrm{Ct}_{\text {gene }}-\mathrm{Ct}_{\text {reference, }}$ with fold changes calculated using the 2- $\triangle \triangle \mathrm{Ct}$ method. GAPDH was used to accomplish the expression normalization.

\section{Plasmids and lentiviruses}

The plasmids pRSV-Rev (12253), pMDLg/pRRE (12251), and pCMV-VSV-G (8454) were purchased from Addgene (Cambridge, MA, USA). The lentiviral vector plko.1 was obtained from Generay Biotech (Shanghai, China). The sequence for p53 shRNA (CCGGGTCCAGATGAAGCTCCCAGAACTCGAGT TCTGGGAGCTTCATCTGGACTTTTT) and flanked by EcoRI and BamHI sites (5' and 3', respectively) were inserted into the plko.1 vector. Viruses were propagated in $293 \mathrm{~T}$ cells, the supernatant of infected 293T cells were collected, and viral particles were pelleted through a $20 \%$ sucrose cushion at $28,000 \mathrm{rpm}$ for $90 \mathrm{~min}$. Purified virions were stored at $-80^{\circ} \mathrm{C}$.

\section{siRNAs}

Synthetic siRNA [negative-control fluorescein (FAM) siRNAs, DR4 siRNAs, and DR5 siRNAs] were obtained from GenePharma (Shanghai, China). The cellular delivery of siRNA was performed using Lipofectamine 2000 (Thermo Fisher Scientific), optimized using various siRNA concentrations, and evaluated by fluorescence microscope and qRT-PCR. siRNAs sequences are listed in Table S2.

\section{Statistical analysis}

Differences among test groups were analyzed by GraphPad Prism software (v7.0; GraphPad Software) and SPSS (v20.0; IBM, Armonk, NY, USA). A P < 0.05 was considered statistically significant. A two-tailed Student's $t$ test was performed to detect statistical difference between two individual experimental groups.

\section{Abbreviations}

Andro: andrographolide; IC50: 50\% inhibitory concentration; MTS: 3-(4,5-dimethylthiazol-2-yl)-5-(3carboxymethoxyphenyl)-2-(4-sulphophenyl)-2H-tetra zolium; TRAIL: tumor necrosis factor-related apoptosis-inducing ligand; BLCA: bladder urothelial carcinoma; TCGA: The Cancer Genome Atlas; MMP: matrix metalloproteinase; DMSO: dimethyl sulfoxide; FITC: fluorescein isothiocyanate; GSEA: Gene set enrichment analysis; Nec-1: necrostatin-1; SD: standard deviation; shRNA: short-hairpin RNA; TNFRSF10A/B: tumor necrosis factor receptor superfamily member 10A/B; Bcl-2: B cell lymphoma 2; cIAP2: cellular inhibitor of apoptosis 2; qRT-PCR: quantitative reverse transcription polymerase chain reaction; RelA: NF-кB p65 subunit; XIAP: X-linked inhibitor of apoptosis.

\section{Supplementary Material}

Supplementary figure and tables. http://www.ijbs.com/v15p0688s1.pdf

\section{Acknowledgments}

We thank Junliang Chang and Yuanyuan Li for their technical assistance and Editage for editorial 
assistance. This work was supported by funding from the National Natural Science Foundation of China (81772183).

\section{Author Contributions}

Conceptualization, Weiwei and Yuyou Deng; Methodology, Yuyou Deng, Haoran Guo and Ran Bi; Software, Yuyou Deng; Validation, Jiaxin Yang; Investigation, Honghuang Lan; Resources, Chunxi Wang; Writing-Review \& Editing, Weiwei and Yuyou Deng; Visualization, Yuyou Deng; Supervision, Chunxi Wang; Project administration, Songling Zahgn; Funding Acquisition, Weiwei and Chunxi Wang.

\section{Competing Interests}

The authors have declared that no competing interest exists.

\section{References}

1. Antoni S, Ferlay J, Soerjomataram I, Znaor A, Jemal A, Bray F. Bladder Cancer Incidence and Mortality: A Global Overview and Recent Trends. European urology. 2017; 71: 96-108

2. Shrader M, Pino M, Lashinger L, Bar-Eli M, Adam L, Dinney C, et al. Gefitinib reverses TRAIL resistance in human bladder cancer cell lines via inhibition of AKT-mediated X-linked inhibitor of apoptosis protein expression. Cancer Res. 2007; 67: 1430-5.

3. Cao JY, Yin HS, Li HS, Yu XQ, Han X. Interleukin-27 augments the inhibitory effects of sorafenib on bladder cancer cells. Brazilian journal of medical and biological research $=$ Revista brasileira de pesquisas medicas e biologicas . 2017; 50: e6207.

4. Mahalingam D, Szegezdi E, Keane M, de Jong S, Samali A. TRAIL receptor signalling and modulation: Are we on the right TRAIL? Cancer Treat Rev. 2009; 35: 280-8.

5. Johnstone R, Frew A, Smyth M. The TRAIL apoptotic pathway in cancer onset, progression and therapy. Nat Rev Cancer. 2008; 8: 782-98.

6. Huang Y, Sheikh MS. TRAIL death receptors and cancer therapeutics. Toxicology and applied pharmacology. 2007; 224: 284-9.

7. Scaffidi C, Schmitz I, Krammer PH, Peter ME. The role of c-FLIP in modulation of CD95-induced apoptosis. The Journal of biological chemistry. 1999; 274: $1541-8$

8. Ashkenazi A. Targeting death and decoy receptors of the tumour-necrosis factor superfamily. Nat Rev Cancer. 2002; 2: 420-30.

9. Smyth M, Takeda K, Hayakawa Y, Peschon J, van den Brink M, Yagita $H$. Nature's TRAIL--on a path to cancer immunotherapy. Immunity. 2003; 18: 1-6.

10. Chen M, Wang X, Zha D, Cai F, Zhang W, He Y, et al. Apigenin potentiates TRAIL therapy of non-small cell lung cancer via upregulating DR4/DR5 expression in a p53-dependent manner. Scientific reports. 2016; 6: 35468.

11. Kang $\mathrm{KH}$, Han $\mathrm{MH}$, Jeong JW, Park $\mathrm{C}$, Lee $\mathrm{SH}$, Lee $\mathrm{HW}$, et al. Bufalin sensitizes human bladder carcinoma cells to TRAIL-mediated apoptosis. Oncology letters. 2017; 14: 853-9.

12. Wang S, El-Deiry WS. TRAIL and apoptosis induction by TNF-family death receptors. Oncogene. 2003; 22: 8628-33

13. Chen S, Fu L, Raja SM, Yue P, Khuri FR, Sun SY. Dissecting the roles of DR4, DR5 and c-FLIP in the regulation of geranylgeranyltransferase I inhibition-mediated augmentation of TRAIL-induced apoptosis. Molecular cancer. 2010; 9: 23

14. Calabrese C, Berman S, Babish J, Ma X, Shinto L, Dorr M, et al. A phase I trial of andrographolide in HIV positive patients and normal volunteers. Phytotherapy research : PTR. 2000; 14: 333-8.

15. Shen YC, Chen CF, Chiou WF. Andrographolide prevents oxygen radical production by human neutrophils: possible mechanism(s) involved in its anti-inflammatory effect. British journal of pharmacology. 2002; 135: 399-406.

16. Liu $\mathrm{SH}$, Lin $\mathrm{CH}$, Liang $\mathrm{FP}$, Chen $\mathrm{PF}$, Kuo $\mathrm{CD}$, Alam $\mathrm{MM}$, et al. Andrographolide downregulates the $\mathrm{v}$-Src and $\mathrm{Bcr}-\mathrm{Abl}$ oncoproteins and induces Hsp90 cleavage in the ROS-dependent suppression of cancer malignancy. Biochemical pharmacology. 2014; 87: 229-42.

17. Zhang C, Qiu X. Andrographolide radiosensitizes human ovarian cancer SKOV3 xenografts due to an enhanced apoptosis and autophagy. Tumour biology : the journal of the International Society for Oncodevelopmental Biology and Medicine. 2015; 36: 8359-65.

18. Dai L, Wang G, Pan W. Andrographolide Inhibits Proliferation and Metastasis of SGC7901 Gastric Cancer Cells. BioMed research international. 2017; 2017 : 6242103.
19. Zhang L, Fang B. Mechanisms of resistance to TRAIL-induced apoptosis in cancer. Cancer gene therapy. 2005; 12: 228-37.

20. Trivedi R, Mishra DP. Trailing TRAIL Resistance: Novel Targets for TRAIL Sensitization in Cancer Cells. Frontiers in oncology. 2015; 5: 69.

21. Ahmed SM, Wu X, Jin X, Zhang X, Togo Y, Suzuki T, et al. Synergistic induction of apoptosis by mapatumumab and anthracyclines in human bladder cancer cells. Oncology reports. 2015; 33: 566-72.

22. Szliszka E, Mazur B, Zydowicz G, Czuba ZP, Krol W. TRAIL-induced apoptosis and expression of death receptor TRAIL-R1 and TRAIL-R2 in bladder cancer cells. Folia histochemica et cytobiologica. 2009; 47: 579-85.

23. Piotrowski WI, Nawrocka-Kunecka A, Antczak A, Marczak J, Biernacki R, Kordek P, et al. Metalloproteinases MMP-9, MMP-2 and their tissue inhibitors TIMP-1, TIMP-2 in peripheral transbronchial lung biopsies of patients with sarcoidosis. Polskie Archiwum Medycyny Wewnetrznej. 2009; 119: 628-35.

24. London CA, Sekhon HS, Arora V, Stein DA, Iversen PL, Devi GR. A novel antisense inhibitor of MMP-9 attenuates angiogenesis, human prostate cancer cell invasion and tumorigenicity. Cancer gene therapy. 2003; 10: 823-32.

25. Chen Z, Sangwan V, Banerjee S, Chugh R, Dudeja V, Vickers SM, et al. Triptolide sensitizes pancreatic cancer cells to TRAIL-induced activation of the death receptor pathway. Cancer letters. 2014; 348: 156-66.

26. Omar H, Arafa e-S, Maghrabi I, Weng J. Sensitization of hepatocellular carcinoma cells to Apo2L/TRAIL by a novel Akt/NF-kB signalling inhibitor. Basic Clin Pharmacol Toxicol. 2014; 114: 464-71.

27. Sanchez-Carbayo M, Socci ND, Lozano JJ, Li W, Charytonowicz E, Belbin TJ, et al. Gene discovery in bladder cancer progression using cDNA microarrays. The American journal of pathology. 2003; 163: 505-16.

28. Wei RJ, Zhang XS, He DL. Andrographolide sensitizes prostate cancer cells to TRAIL-induced apoptosis. Asian journal of andrology. 2017.

29. Zhou J, Lu GD, Ong CS, Ong CN, Shen HM. Andrographolide sensitizes cancer cells to TRAIL-induced apoptosis via p53-mediated death receptor 4 up-regulation. Molecular cancer therapeutics. 2008; 7: 2170-80.

30. Lim SC, Jeon HJ, Kee KH, Lee MJ, Hong R, Han SI. Andrographolide induces apoptotic and non-apoptotic death and enhances tumor necrosis factor-related apoptosis-inducing ligand-mediated apoptosis in gastric cancer cells. Oncology letters. 2017; 13: 3837-44.

31. Xia Y, Shen S, Verma IM. NF-kappaB, an active player in human cancers. Cancer immunology research. 2014; 2: 823-30.

32. Chen X, Kandasamy K, Srivastava R. Differential roles of RelA (p65) and c-Rel subunits of nuclear factor kappa B in tumor necrosis factor-related apoptosis-inducing ligand signaling. Cancer Res. 2003; 63: 1059-66.

33. Zhang R, Zhao J, Xu J, Jiao DX, Wang J, Gong ZQ et al. Andrographolide suppresses proliferation of human colon cancer SW620 cells through the TLR4/NF-kappaB/MMP-9 signaling pathway. Oncology letters. 2017; 14: 4305-10.

34. Zhang Q, Ding Y, Lei Y, Qi C, He X, Lan T, et al. Andrographolide suppress tumor growth by inhibiting TLR4/NF-KB signaling activation in insulinoma. Int J Biol Sci. 2014; 10: 404-14.

35. Sanchez-Carbayo M, Socci ND, Lozano J, Saint F, Cordon-Cardo C. Defining molecular profiles of poor outcome in patients with invasive bladder cancer using oligonucleotide microarrays. Journal of clinical oncology : official journal of the American Society of Clinical Oncology. 2006; 24: 778-89.

36. Subramanian A, Tamayo P, Mootha VK, Mukherjee S, Ebert BL, Gillette MA et al. Gene set enrichment analysis: a knowledge-based approach for interpreting genome-wide expression profiles. Proceedings of the National Academy of Sciences of the United States of America. 2005; 102: 15545-50. 